\title{
New Independent Thermobalancing Treatment with Therapeutic Device for Chronic Prostatitis/Chronic Pelvic Pain Syndrome
}

\author{
Simon Allen, ${ }^{1, *}$ and Ivan Gerasimovich Aghajanyan ${ }^{2}$ \\ ${ }^{1} \mathrm{MD}$, PhD, Director, Fine Treatment, Oxford, United Kingdom \\ ${ }^{2}$ Prof, MD, PhD Head, Department of Urology, Yerevan State Medical University, Yerevan, Armenia \\ "Corresponding author: Simon Allen, MD, PhD, Director, Fine Treatment, Oxford, United Kingdom, E-mail: info@finetreatment.com
}

Received 2016 December 11; Accepted 2017 January 07.

\begin{abstract}
Background: Medications, alternative and complementary treatments for type-III chronic prostatitis/chronic pelvic pain syndrome $(\mathrm{CP} / \mathrm{CPPS})$ are used frequently. The aim of this article is to define thermobalancing therapy as an independent treatment for internal diseases, such as $\mathrm{CP} / \mathrm{CPPS}$.

Methods: The effect of thermobalancing therapy (TT) by using Dr. Allen's therapeutic device-(DATD) on patients with CP/CPPS was investigated. National institute of health chronic prostatitis symptom index (NIH-CPSI) scores, prostatic volume(PV), and maximum urinary flow rate $\left(\mathrm{Q}_{\max }\right)$ were measured in one group of 45 patients who underwent TT and a control group that did not have TT. These all parameters were compared between groups.

Results: No significant difference was found at baseline evaluation in treatment and control groups with regard to age, NIH-CPSI score, $\mathrm{PV}$ or $\mathrm{Q}_{\max }$. In the treatment group pain score decreased and quality of life(QoL) improved significantly, whereas in the control group no changes. TT reduced PV and increased $\mathrm{Q}_{\max }$ significantly, whereas in the control group TT did not elicit significant changes in $P V$ and $Q_{\max }$.

Conclusions: The study has explored that TT with DATD as monotherapy for CP/CPPS patients: (i) reduces pain dramatically and improves QoL; (ii) reduces PV and increases $\mathrm{Q}_{\max }$. None of the patients who received TT suffered side effects and the cost of TT compares favourably with the cost of conventional treatment. Thus, TT could be recommended as a new independent treatment for CP/CPPS.
\end{abstract}

Keywords: Chronic Prostatitis, Chronic Pelvic Pain Syndrome, Thermobalancing Therapy, Prostate Gland, Alternative Treatment, Therapeutic Device

\section{Background}

Chronic internal non-infectious diseases are hard to treat and chronic prostatitis is one of them. Among middle-aged men, prostatitis/chronic pelvic pain syndrome (CP/CPPS) is the leading cause of urological counseling and outpatient clinic visits (1). As young and middleaged men are more likely to develop chronic prostatitis/chronic pelvic pain syndrome (CP/CPPS) the use of surgical procedures is not recommended (2). Therefore as a prostate cannot be operated and there are no drugs that can reach this organ easily, it is a common view that patients with CP/CPPS should get phenotype drive therapy(3).

The lack of effective medical therapy for CP/CPPS, like nonsteriodal anti-inflammatories, alpha-blockers, antimicrobial therapy, and 5a-reductase inhibitors, has led to various attempts to use alternative therapies, phytotherapy, cannabis, acupuncture, physical therapy, and psychosocial factors $(4,5)$. Spicy food, coffee, alcoholic beverages and strong tea can exacerbate the symptoms CP/CPPS while other nutrients, such as psyllium, water, herbal teas, and polycarbophil, can improve condition of patients with this disorder (6). Eating habits such as "frequency of eating fast food" may lead to significant negative impact on the symptoms of CP/CPPS (7). However, there is no association has been found between major lifestyle factors (obesity, smoking and hypertension) and CP/CPPS disease risk (8).

Chinese herbal medicine for chronic prostatitis is used frequently and more importantly this mono-therapy is safe (9). Shoskes (10) in his review in 2002 has noticed that among plentiful herbal treatments the promising include zinc, cernilton (bee pollen), quercetin, and saw palmetto. About $69 \%$ of pollen extract patients showed a $25 \%$ improvement compared to $48.5 \%$ of placebo patients has shown the clinical trial published in 2009 (11). Thus, pollen extracts (Cernilton) compares favorably to other phytotherapeutics agents: quercetin, and saw palmetto (Serenoa repens) (12).

Physical therapies for $\mathrm{CP} / \mathrm{CPPS}$ have shown that patients responded to myofascial physical therapy (MPT) and global therapeutic massage (GTM), 57\% vs. $21 \%$ accordingly. Furthermore it was noticed that men with CP/CPPS responded in a more positive fashion to GTM provided by female physical therapists (13). Different psychosocial 
factors may play substantial role on men with CP/CPPS, such as stress, personality factors, social aspects as well as different psychiatric co-morbidities (depression, anxiety and trauma-related disorders, somatization disorder, substance abuse); and affect their quality of life - QoL (14).

Thermobalancing therapy (TT) enabled by physiotherapeutic device has demonstrated improvement of QoL in men with CP/CPPS (15). TT has been implemented for termination of "micro-focus" of hypothermia following the constriction and spontaneous expansion of capillaries in the prostate tissue, i.e., the main trigger of chronic prostatitis. This described changes lead to increased pressure in the prostate gland, thereby causing the symptoms of CP/CPPS (16).

There were reported positive outcomes after acupuncture that can decrease pain, improve quality of life and potentially modulate inflammation (17). The review of published randomized control trials that compared the efficacy of acupuncture with sham acupuncture or standard medical treatment for patients with $\mathrm{CP} / \mathrm{CPPS}$ observed that it has better efficacy (18). However different studies have found specific effects associated with acupuncture last at least over the short term, so acupuncture has relatively short-term physiological effects $(19,20)$.

Dr. Allen's therapeutic device (DATD) that provides TT has shown its efficacy in men with lower urinary tract symptoms (LUTS) and benign prostatic hyperplasia decreasing prostate volume and LUTS by improving blood circulation in prostate $(21,22)$.

\section{Objectives}

The aim of this study is to confirm that TT is a unique independent treatment for CP/CPPS by assessing the dynamics of pain and QoL, PV, and $\mathrm{Q}_{\max }$.

\section{Methods}

\subsection{Ethical Approval of the Study Protocol}

The ethics committee of Yerevan State Medical University (Yerevan, Armenia) approved the study protocol. All participants provided written informed consent to be included in the study.

\subsection{Study Design}

This was an observational clinical controlled study undertaken in 2013 - 2015 that measured national institute of health chronic prostatitis symptom index (NIH-CPSI) scores for pain and quality of life (QoL), prostate volume $(\mathrm{PV})$, and maximum urinary flow rate $\left(\mathrm{Q}_{\max }\right)$ in 6-month, in one group of 45 patients who underwent TT and a control group of 45 patients that did not have such treatment, and compared these parameters between the two groups.

\subsection{Participants}

Over a 2-year period, 45 males (age $<55$ years) with $\mathrm{CP} / \mathrm{CPPS}$ (NIH category III) were selected for a clinical trial. Patients were selected in conjunction with urologists. Inclusion criteria were men with CP and NIH-CPSI pain score $\geq 1$. Exclusion criteria were acute prostatitis, urethral stricture, neurogenic bladder, and comorbidities (e.g., impaired renal function, diabetes mellitus). We included patients with levels of prostate-specific antigen (PSA) in serum $>4 \mathrm{ng} / \mathrm{mL}$ for whom biopsy of the prostate gland showed no evidence of cancer. Medical treatments at the time of study enrolment were not taken into consideration.

\subsection{Evaluation}

Effectiveness of TT was studied by comparing men with $\mathrm{CP} / \mathrm{CPPS}$ who received DATD as monotherapy with the control group (who received no treatment). Baseline evaluations comprised complete physical examination, medical history, digital rectal examination, serum biochemistry, PSA measurement, electrolyte levels, urinalysis and renal function tests. Evaluations were made at baseline and 6 months after treatment. At baseline assessment, patients were evaluated for various scores (NIH-CPSI pain/QoL), PV $(\mathrm{mL})$ and uroflowmetry $\left(\mathrm{Q}_{\max } ; \mathrm{mL} / \mathrm{s}\right)$.

Dynamics of the symptoms and indicators in each group were assessed at the beginning and end of treatment using NIH-CPSI scores. Uroflowmetry was undertaken using a Sanuro2UL system (Santron Meditronic, Maharashtra, India). Ultrasound was used to determine the volume of the prostate gland by employing a US9000E2 ultrasound scanner (Rising Medical Equipment, Beijing, China). The standard ellipsoid formula length $x$ width $\times$ height $\times 0.52$ was used to determine PV.

\subsection{Statistical Analyses}

The independent-samples t-test and paired-samples ttest are suitable only for interval and ratio data, so the Wilcoxon signed-rank test was employed. $\mathrm{P}<0.05$ was considered significant. Statistical analyses were carried out using SPSS v22 (IBM, Armonk, NY, USA).

\subsection{Endpoints}

Primary endpoints were changes in pain score and QoL score after 6 months of treatment. Secondary endpoints were changes in $\mathrm{PV}$ and $\mathrm{Q}_{\max }$ after 6 months of treatment. 


\subsection{Thermobalancing Natural Treatment With DATD}

DATD provides a method of treating an affected prostate gland by application of a special mixture of waxes ("thermoelement") topically upon its projection. This thermoelement can accumulate the emitted body heat, and thus turn into a source of energy itself. In this way, DATD applies the thermoelement tightly to the coccyx, thereby overcoming the skin barrier and spreading heat energy towards the prostate gland (Figure 1).

Figure 1. The Device Tightly Applies a Natural Thermoelement to the Coccyx Area Allowing Accumulation of Body Heat

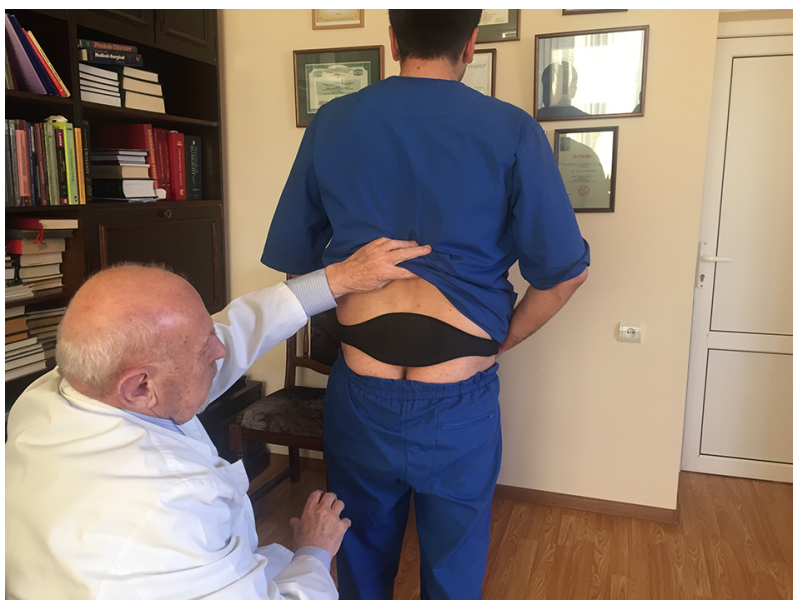

It acts as the heat source for the prostate.

Men who met the inclusion criteria of the study had treatment for CP/CPPS using DATD. In April 2010, DATD was registered at the Medicines and Healthcare Products Regulatory Agency as a class-1 medical device $(23,24)$.

\section{Results}

\subsection{Pain Score and QoL}

In the treatment group, the mean pain score decreased from $10.38 \pm 2.53$ to $3.58 \pm 2.54$ by the end of the study period at a significance level of $\mathrm{P}<0.001$. In the control group, the mean pain score decreased from $10.49 \pm 2.46$ to $9.71 \pm 2$, so slightly over the same period. Hence, there was a significant decrease in pain in the treatment group, whereas in the control group pain score decreased slightly.

In the treatment group, the mean QoL decreased from $8.11 \pm 2.00$ to $2.98 \pm 1.80$ at a significance level of $\mathrm{P}<0.001$. In the control group QoL score decreased slightly from 8.47 \pm 1.92 to $8.33 \pm 1.72$. These results suggested that TT improved QoL significantly, whereas in the control group no changes in QoL.

\subsection{PV and Qmax}

In the treatment group PV mL decreased from $31.75 \pm$ 7 to $27.07 \pm 4.52$ at a significance level of $\mathrm{P}<0.001$. In the control group PV mL increased from $30.77 \pm 6,43$ to 31.58 $\pm 7.13 \mathrm{~mL}$ at the end of the study period. These data suggest that TT reduced PV significantly whereas, in the control group, PV increased.

In the treatment group $\mathrm{Q}_{\max } \mathrm{mL} / \mathrm{s}$, increased from 11.93 \pm 4.344 to $16.45 \pm 3.50 \mathrm{~mL} / \mathrm{s}$ at a significance level of $\mathrm{P}<$ 0.001. In the control group $\mathrm{Q}_{\max }$ decreased from $12.59 \pm$ 3.57 to $12.20 \pm 2.54 \mathrm{~mL} / \mathrm{s}$. These results suggest that TT increases $\mathrm{Q}_{\max }$ significantly in $\mathrm{CP} / \mathrm{CPPS}$ patients whereas, in the control group no changes in $\mathrm{Q}_{\max }$.

\subsection{Side Effects TT and DATD}

Side effects of TT with DATD were not observed in any patient.

\section{Discussion}

The present study showed that 6-month natural TT with DATD: (i) reduces pain and improves QoL; (ii) reduces PV and increases $\mathrm{Q}_{\max }$. We believe that TT is effective an independent treatment for $\mathrm{CP} / \mathrm{CPPS}$ because treats the underlying cause of this chronic disease. DADT gradually reduces the size of inflamed prostate gland improving its functioning, decreases disturbing symptoms and increases urinary flow. Clinical improvement, including practical changes in the ultrasound/uroflowmetry parameters in patients with $\mathrm{CP} / \mathrm{CPPS}$ who used DATD could be explained by changes in blood circulation at the capillary level. The continuous heat exposure from thermoelement that does not exceed the normal body temperature has terminated micro-focus of hypothermia and spontaneous expansion of capillaries. The similar positive effect has been seen while clinical trial on TT in men with enlarged prostate (25). DATD removes the vicious cycle of spontaneous growth of capillaries, thereby relieving pressure in the prostate gland and the CP/CPPS symptoms (26).

This clinical trial was not randomized because most men with CP/CPPS had psychological problems. Indeed, some scholars believe that psychological problems are a component of CP/CPPS (27). Blinding of patients/assessors to the study protocol was not possible because DATD had to be applied to participants and measurements taken. Thus this study was not a randomized. Having a "placebo" or "sham" group as controls could have provided more statistical rigor with regard to outcomes. However, in men with $\mathrm{CP} / \mathrm{CPPS}$ largely unresponsive to medical interventions, greater pain is associated with diminished QoL (28). Therefore, proposing that men with $\mathrm{CP} / \mathrm{CPPS}$ should wear 


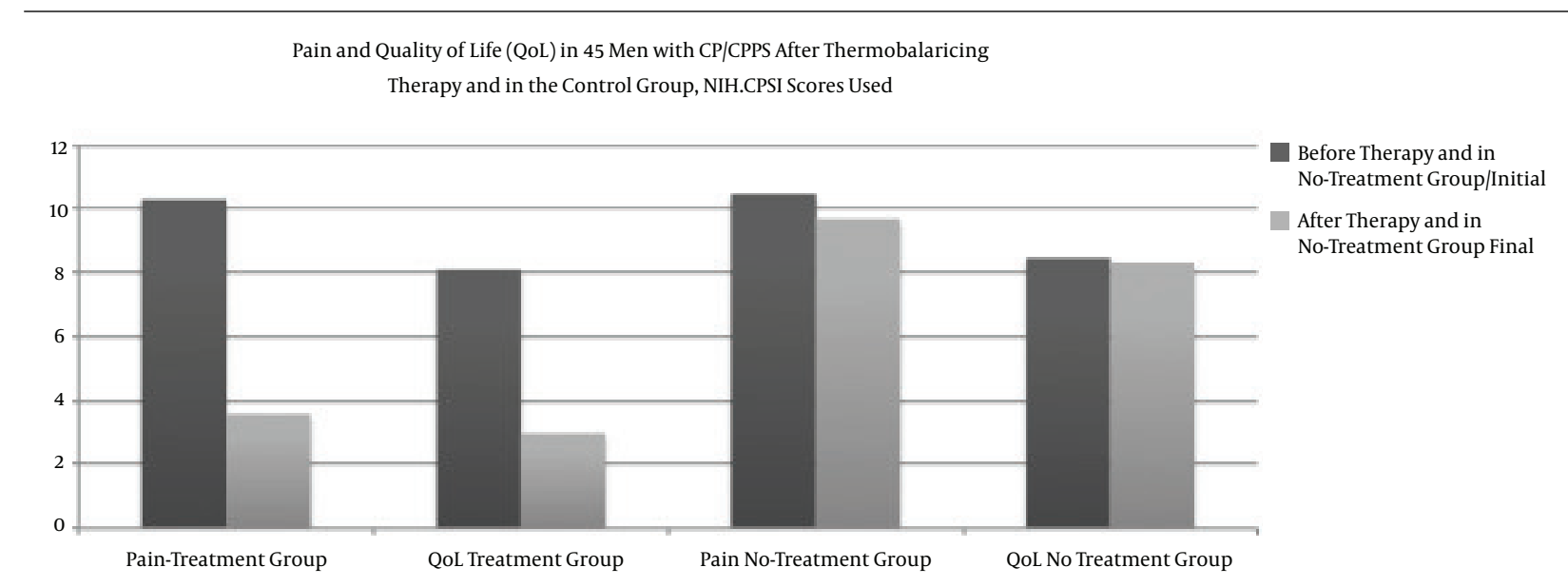

Figure 2. Dynamics of Pain and Quality of Life Scores in 45 Men With CP/CPPS After Thermobalancing Therapy and in the Control Group Measured by the National Institute of Health-Chronic Prostatitis Symptom Index (NIH-CPSI)

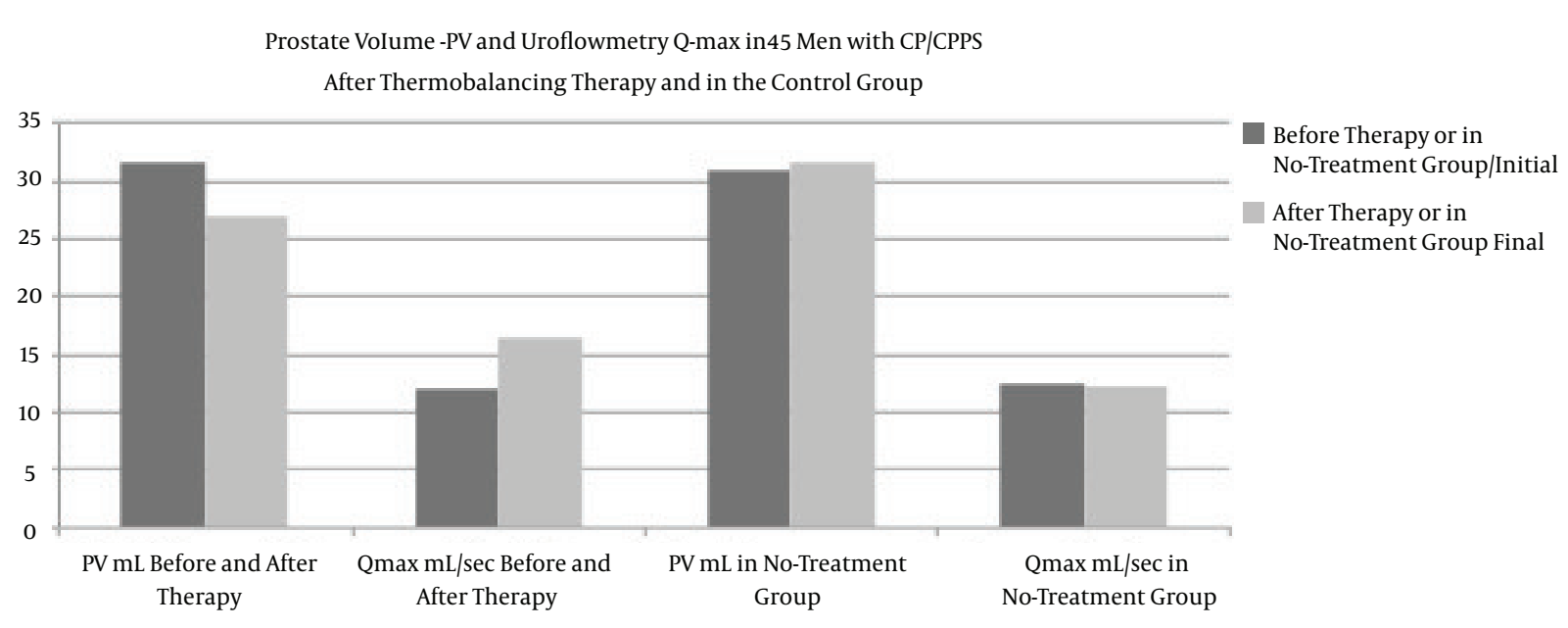

Figure 3. Prostate Volume (PV mL) and Maximum Urinary Flow Rate $\left(\mathrm{Q}_{\max } \mathrm{mL} / \mathrm{sec}\right)$ in $45 \mathrm{Men}$ With CP/CPPS After Thermobalancing Therapy and in the Control Group Measured by Ultrasound and Uroflowmetry Accordingly

a "placebo belt" for 6 months that does not alleviate symptoms would be very difficult. Usually, patients with CP felt symptom relief within weeks of wearing DATD, and used the device as required.

Physicians currently prescribe a wide range of medications, some of which have studied, and numerous of them have not been studied yet in randomized controlled trials (RCTs), however, none of them has shown high efficacy (29). Many new drugs for CP/CPPS treatment were found to be valueless $(30,31)$, and most commonly used drugs have adverse effects, especially when used in longterm (32). Zhang with colleagues investigated associations between lifestyle factors and CP/CPPS on a large number of men. However, no associations were observed between ma- jor lifestyle factors (obesity, smoking, and hypertension) and CP/CPPS (8). There is poor evidence supporting the efficacy of physical treatment options for CP/CPPS that include repetitive prostate massage or methods to apply energy to the prostate, so they remain controversial (33). Some researches reveal effectiveness of acupuncture for $\mathrm{CP} / \mathrm{CPPS}$ and recommend applying it for managing pains of $\mathrm{CP} / \mathrm{CPPS}$ with standard treatment options (34). However, there is no evidence for how long the effect after acupuncture lasts.

It should be noted that, contrary to conventional and alternative treatment options for CP/CPPS, TT stimulates the processes in prostate for prolong period of time, providing the treatment of its cause (35). The use of TT using DATD does not impede men's day-to-day activity. Impor- 
tantly, none of the patients who received TT suffered side effects and the cost of DATD compares favourably with the cost of conventional treatment. The present study shows effectiveness of new natural therapy for CP/CPPS which can be recommended as a new independent treatment for this chronic condition.

\subsection{Conclusions}

Six-month clinical study confirmed the ability of TT with DATD as a montherapy to reduce PV and pain, and improve QoL in men with CP/CPPS, therefore, this natural therapy should be recommended as an independent and traditional treatment option for these patients. The effectiveness of this therapy is in its capacity to influence the etiology of $\mathrm{CP} / \mathrm{CPPS}$.

\section{Acknowledgments}

We are grateful to the staff of the department of urology of the Yerevan State Medical University and the Mikaelyan institute of surgery (Yerevan, Armenia) for their help in supervision of patients during the study.

\section{Footnote}

Funding/Support: $S$ Allen is an author of US patent, US 9,408,744 B2, on therapeutic device and method i.e. Thermobalancing therapy and therapeutic device. United States patent and trademark office, USPTO have issued the patent, in August 2016. Both authors have not received reimbursements, fees, funding, or salary relating to the content of this manuscript.

\section{References}

1. Chung SD, Huang CC, Lin HC. Chronic prostatitis and depressive disorder: a three year population-based study. J Affect Disord. 2011;134(13):404-9. doi: 10.1016/j.jad.2011.05.046. [PubMed: 21665291].

2. Engeler DS, Baranowski AP, Dinis-Oliveira P, Elneil S, Hughes J, Messelink EJ, et al. The 2013 EAU guidelines on chronic pelvic pain: is management of chronic pelvic pain a habit, a philosophy, or a science? 10 years of development. Eur Urol. 2013;64(3):431-9. doi: 10.1016/j.eururo.2013.04.035. [PubMed: 23684447].

3. Polackwich AS, Shoskes DA. Chronic prostatitis/chronic pelvic pain syndrome: a review of evaluation and therapy. Prostate Cancer Prostatic Dis. 2016;19(2):132-8. doi: 10.1038/pcan.2016.8. [PubMed: 26951713].

4. Herati AS, Moldwin RM. Alternative therapies in the management of chronic prostatitis/chronic pelvic pain syndrome. World J Urol. 2013;31(4):761-6. doi: 10.1007/s00345-013-1097-0. [PubMed: 23740129].

5. Tripp DA, Nickel JC, Katz L, Krsmanovic A, Ware MA, Santor D. A survey of cannabis (marijuana) use and self-reported benefit in men with chronic prostatitis/chronic pelvic pain syndrome. Can Urol Assoc J. 2014;8(11-12):901-5. doi: 10.5489/cuaj.2268.
6. Herati AS, Shorter B, Srinivasan AK, Tai J, Seideman C, Lesser M, et al. Effects of foods and beverages on the symptoms of chronic prostatitis/chronic pelvic pain syndrome. Urology. 2013;82(6):1376-80. doi: 10.1016/j.urology.2013.07.015. [PubMed: 23978369].

7. Wang Y, Chen C, Zhu C, Chen L, Han Q, Ye H. Social Determinants of Chronic Prostatitis/Chronic Pelvic Pain Syndrome Related Lifestyle and Behaviors among Urban Men in China: A Case-Control Study. Biomed Res Int. 2016;2016 doi: 10.1155/2016/1687623.

8. Zhang R, Sutcliffe S, Giovannucci E, Willett WC, Platz EA, Rosner BA, et al. Lifestyle and Risk of Chronic Prostatitis/Chronic Pelvic Pain Syndrome in a Cohort of United States Male Health Professionals. J Urol. 2015;194(5):1295-300. doi: 10.1016/j.juro.2015.05.100. [PubMed: 26070893].

9. Wang Z, Yuan L, Wang Y, Yang B, Dong X, Gao Z. Efficacy and safety of Chinese herbal medicine for chronic prostatitis associated with damp-heat and blood-stasis syndromes: a meta-analysis and literature review. Patient Prefer Adherence. 2016;10:1889-902. doi: 10.2147/PPA.S108699. [PubMed: 27698555]

10. Shoskes DA. Phytotherapy in chronic prostatitis. Urology. 2002;60(6 Suppl):35-7. doi: 10.1016/S0090-4295(02)02383-X. [PubMed: 12521591].

11. Wagenlehner FM, Schneider H, Ludwig M, Schnitker J, Brahler E, Weidner W. A pollen extract (Cernilton) in patients with inflammatory chronic prostatitis-chronic pelvic pain syndrome: a multicentre, randomised, prospective, double-blind, placebo-controlled phase 3 study. Eur Urol. 2009;56(3):544-51. doi: 10.1016/j.eururo.2009.05.046. [PubMed: 19524353].

12. Tandogdu Z, Cek M. The Role of Phytotherapy in the Management of Prostatitis Patients. UK: Springer; 2016. pp. 61-5.

13. Fitzgerald MP, Anderson RU, Potts J, Payne CK, Peters KM, Clemens JQ, et al. Randomized multicenter feasibility trial of myofascial physical therapy for the treatment of urological chronic pelvic pain syndromes. J Urol. 2013;189(1 Suppl):75-85. doi: 10.1016/j.juro.2012.11.018. [PubMed: 23234638].

14. Riegel B, Bruenahl CA, Ahyai S, Bingel U, Fisch M, Lowe B. Assessing psychological factors, social aspects and psychiatric co-morbidity associated with Chronic Prostatitis/Chronic Pelvic Pain Syndrome(CP/CPPS) in men - a systematic review. J Psychosom Res. 2014;77(5):333-50. doi: 10.1016/j.jpsychores.2014.09.012. [PubMed: 25300538]

15. Aghajanyan IG, Allen S. Positive Response to Thermobalancing Therapy Enabled by Therapeutic Device in Men with Non-Malignant Prostate Diseases. BPH Chronic Prostatitis, Dis. 2016;4:18. doi: 10.3390/diseases4020018.

16. Allen S, Adjani A. Therapeutic Device and Method, United States Patent and Trademark Office 2016. Available from: https://www. google.com/patents/US9408744.

17. Sahin S, Bicer M, Eren GA, Tas S, Tugcu V, Tasci AI, et al. Acupuncture relieves symptoms in chronic prostatitis/chronic pelvic pain syndrome: a randomized, sham-controlled trial. Prostate Cancer Prostatic Dis. 2015;18(3):249-54. doi: 10.1038/pcan.2015.13. [PubMed: 25939517].

18. Chang SC, Hsu CH, Hsu CK, Yang SS, Chang SJ. The efficacy of acupuncture in managing patients with chronic prostatitis/chronic pelvic pain syndrome: A systemic review and meta-analysis. Neurourol Urodyn. 2016 doi: 10.1002/nau.22958. [PubMed: 26741647].

19. Qin Z, Wu J, Zhou J, Liu Z. Systematic Review of Acupuncture for Chronic Prostatitis/Chronic Pelvic Pain Syndrome. Medicine (Baltimore). 2016;95(11):3095. doi: 10.1097/MD.0000000000003095. [PubMed: 26986148].

20. Cherkin DC, Sherman KJ, Hogeboom CJ, Erro JH, Barlow WE, Deyo RA, et al. Efficacy of acupuncture for chronic low back pain: protocol for a randomized controlled trial. Trials. 2008;9:10. doi:10.1186/1745-62159-10. [PubMed: 18307808].

21. Allen S, Aghajanyan IG. Thermobalancing conservative treatment for moderate-to-low-degree lower urinary tract symptoms (LUTS) secondary to prostate enlargement. Cogent Medicine. 2016;3(1):1195067. doi: 10.1080/2331205X.2016.1195067. 
22. Allen S, Aghajanyan IG. Benign Prostatic Hyperplasia Treatment with New Physiotherapeutic Device. Urol J. 2015;12(5):2371-6. [PubMed: 26571324].

23. CE Marking Consulting Service European Authorized Representative . Class I medical devices, 1996-2015 2016. Available from: http://www. ce-marking.com/medical-devices-class-i.html.

24. Lamph S. Regulation of medical devices outside the European Union. J R Soc Med. 2012;105 Suppl 1:12-21. doi: 10.1258/jrsm.2012.120037. [PubMed: 22508968].

25. Allen S, Aghajanyan I. Use of thermobalancing therapy in ageing male with benign prostatic hyperplasia with a focus on etiology and pathophysiology. Aging Male. 2016:1-5. doi: 10.1080/13685538.2016.1247151. [PubMed: 27960590].

26. Allen S, Aghajanyan I. Effect of thermobalancing therapy on chronic prostatitis and chronic pelvic pain syndrome. J Clin Urol. 2016;20:1-8. doi: 10.1177/2051415816671036.

27. Chung SD, Lin HC. Association between chronic prostatitis/chronic pelvic pain syndrome and anxiety disorder: a population-based study. PLoS One. 2013;8(5):64630. doi: 10.1371/journal.pone.0064630. [PubMed: 23691256].

28. Krsmanovic A, Tripp DA, Nickel JC, Shoskes DA, Pontari M, Litwin MS, et al. Psychosocial mechanisms of the pain and quality of life relationship for chronic prostatitis/chronic pelvic pain syndrome (CP/CPPS). Can Urol Assoc J. 2014;8(11-12):403-8. doi: 10.5489/cuaj.2179. [PubMed:
25553153].

29. Strauss AC, Dimitrakov JD. New treatments for chronic prostatitis/chronic pelvic pain syndrome. Nat Rev Urol. 2010;7(3):127-35. doi 10.1038/nrurol.2010.4. [PubMed: 20142810].

30. Nickel JC, Atkinson G, Krieger JN, Mills IW, Pontari M, Shoskes DA, et al. Preliminary assessment of safety and efficacy in proof-ofconcept, randomized clinical trial of tanezumab for chronic prostatitis/chronic pelvic pain syndrome. Urology. 2012;80(5):1105-10. doi 10.1016/j.urology.2012.07.035. [PubMed: 23010344].

31. Nickel JC, Shoskes DA, Wagenlehner FM. Management of chronic prostatitis/chronic pelvic pain syndrome (CP/CPPS): the studies, the evidence, and the impact. World J Urol. 2013;31(4):747-53. doi 10.1007/s00345-013-1062-y.

32. Nickel JC. Treatment of chronic prostatitis/chronic pelvic pain syndrome. Int J Antimicrob Agents. 2008;31(1):112-6.

33. Lee KS, Choi JD. Chronic prostatitis: approaches for best management. Korean J Urol. 2012;53(2):69-77. doi: 10.4111/kju.2012.53.2.69. [PubMed: 22379583].

34. Lee SH, Lee BC. Use of acupuncture as a treatment method for chronic prostatitis/chronic pelvic pain syndromes. Curr Urol Rep. 2011;12(4):288-96. doi: 10.1007/s11934-011-0186-0. [PubMed: 21472420].

35. Allen S. The Origin of Chronic Diseases Can Be in Capillary Pathology: An Evidence from Clinical Trials on Thermobalancing Treatment of Prostate Reveals. Achiev Life Sci. 2016;9(2) doi:10.1016/j.als.2016.11.005 